\title{
Decision making under time pressure: An independent test of sequential sampling models
}

\author{
ITIEL E. DROR \\ Southampton University, Highfield Southampton, England \\ JEROME R. BUSEMEYER \\ Indiana University, Bloomington, Indiana \\ and \\ BETH BASOLA \\ Southampton University, Highfield Southampton, England
}

\begin{abstract}
Choice probability and choice response time data from a risk-taking decision-making task were compared with predictions made by a sequential sampling model. The behavioral data, consistent with the model, showed that participants were less likely to take an action as risk levels increased, and that time pressure did not have a uniform effect on choice probability. Under time pressure, participants were more conservative at the lower risk levels but were more prone to take risks at the higher levels of risk. This crossover interaction reflected a reduction of the threshold within a single decision strategy rather than a switching of decision strategies. Response time data, as predicted by the model, showed that participants took more time to make decisions at the moderate risk levels and that time pressure reduced response time across all risk levels, but particularly at the those risk levels that took longer time with no pressure. Finally, response time data were used to rule out the hypothesis that time pressure effects could be explained by a fast-guess strategy.
\end{abstract}

Decision making is an inseparable component of all the activities commonly studied by cognitive psychologists. This is manifested by the prominent role that decision processes play in many areas of cognition, ranging from perceptual processes (Link, 1992) to memory recognition (Ratcliff, 1978) and categorization (Nosofsky \& Palmeri, 1997). Recently, a convergence of ideas has formed regarding the dynamic nature of the decision process that underlies all the above-mentioned cognitive abilities (cf. Ratcliff, Van Zandt, \& McKoon, in press)-namely, the idea that activation for or against each action is sequentially sampled, from moment to moment, and accumulated over time. The accumulation process continues until a threshold is reached, at which time a "winner takes all" action is triggered. This sequential sampling type of dynamic decision process has been highly successful in explaining the effects of time pressure on accuracy and speed-accuracy tradeoff (see Link, 1992; Nosofsky \& Palmeri, 1997; Ratcliff et al., in press).

The broad success of sequential sampling models in perception, memory, and categorization has led some de-

This research was supported by grants funded by AFRL (awarded to the first author) and by NSF Grant SBR-9602102 and NIMH Grant 5R01MH55680 (awarded to the second author). We thank Zhengping Ma for help with some of the data analyses and Donna Stevens for proofreading the paper. The authors thank the reviewers for very helpful comments. Correspondence concerning this article should be addressed to I. E. Dror, Department of Psychology, Southampton University, Highfield Southampton SO17 1BJ, England (e-mail: dror@cogito. psy.soton.ac.uk). www: http://www.cogsci.soton.ac.uk/ dror cision researchers to explore their usefulness in the more traditional domain of decision-making tasks, such as risktaking decisions (Albert, Aschenbrenner, \& Schmalhofer, 1989; Aschenbrenner, Albert, \& Schmalhofer, 1986; Busemeyer, 1985; Diederich, 1995; Kornbrot, 1989; Petrusic \& Jamieson, 1978; Wallsten, 1995). However, the majority of decision researchers have not followed this lead, and most of the studies in decision making have centered on testing static models originating from economic rather than cognitive theory (see Goldstein \& Weber, 1996). Consequently, there has been a neglect in conducting independent tests of the predictions made by sequential sampling models of risk-taking decisions. The purpose of this article is to test some basic predictions regarding the effects of time pressure on risk-taking decision making.

The risk-taking decision task used for this experiment was developed by Dror, Katona, and Mungur (1998) and is a simplified variant of the game of blackjack. In this task, the decision maker must decide whether or not to gamble by taking another card from a deck in order to maximize his or her total points without exceeding 21. This task was chosen because the information-processing demands (stimulus encoding and response production) are minimal and identical across trials, thus enabling us to isolate the time pressure effects in the decision stage. Furthermore, this task allowed us to systematically manipulate the levels of risk by varying the probability that taking a card would "bust" (exceed 21).

For this simple task, sequential sampling models make an a priori prediction regarding the effect of time pressure 
on the frequency of choosing the gamble (details are presented in the Appendix): Time pressure will increase the frequency of choosing a gamble when the risk (probability of losing) is high but will decrease the frequency of choosing the gamble when the risk (probability of losing) is low. In other words, sequential sampling models predict a crossover interaction effect between time pressure and risk level on frequency of choosing the gamble. Thus, time pressure is predicted not to have a uniform effect (of being either more conservative or more prone to take risks) but, rather, a polarization in terms of behavior: At the low risk, people turn more conservative and take fewer gambles, whereas at the high risk, they are more risky and take more gambles.

The sequential sampling model also makes specific predictions for this task concerning the mean response times for two different types of responses. A congruent response occurs if a card is chosen when there is very low risk (the response is compatible with the risk level); an incongruent response occurs if a card is not chosen when there is very low risk (the response is not compatible with the risk level). The sequential sampling model predicts that incongruent responses take a longer time to make than congruent responses (see the Appendix for details).

A second reason for selecting this task is that a number of studies have investigated the blackjack game, identifying optimal versus heuristic strategies (Anderson \& Brown, 1984; Bond, 1974; Keren \& Wagenaar, 1985; Phillips \& Amrhein, 1989). The optimal strategy for maximizing the probability of winning is to take an additional card if the current total falls below an optimal cutoff criterion, where this criterion is determined in a complex way that takes into account the card shown for the opposing player. A common heuristic strategy for this task is the never-bust strategy, which is a conservative tendency to avoid taking an additional card and to stay with the current total. Although nonoptimal, the never-bust strategy is attractive because it avoids the possibility of actively taking an action that may be directly responsible for losing the game.

Changes from optimal toward simpler heuristic strategies under time pressure provide an alternative explanation for the effects of time pressure on risk taking (see, e.g., Johnson, Payne, \& Bettman, 1995; Payne, Bettman, $\&$ Luce, 1996). However, the predictions inferred from strategy switching differ from the predictions derived from sequential sampling models for this particular task. It is predicted that, in switching to a never-bust strategy under time pressure, the frequency of choosing the gamble never increases (because the likelihood of switching to the simpler never-bust strategy increases under time pressure).

Another plausible hypothesis is that participants switch to a fast-guess strategy under time pressure. That is, an individual will occasionally make fast random guesses when under time pressure. Like the sequential sampling model, this fast-guess hypothesis predicts a crossover in- teraction for choice probability. But unlike the sequential sampling model, the fast-guess model predicts faster responses for incongruent than for congruent responses (see the Appendix for details).

The study reported here includes two parts. The first part reports the main empirical results obtained from collecting behavioral data on decision making under time pressure. To achieve this goal, we tested participants on Dror et al.'s (1998) risk-taking decision-making task under a time pressure condition and under a no time pressure condition. This was done independently of the second part, which consisted of generating predictions for both choice probability and decision time through the use of a sequential sampling model (Busemeyer \& Townsend, 1993) and comparing the predictions to the actual behavioral data. In the Discussion section, we review related research on decision making under time pressure and contrast the predictions of sequential sampling models with alternative explanations based on changes in heuristic strategies under time pressure.

\section{METHOD}

\section{Participants}

Thirty-two participants (16 males, 16 females) took part in the study for credit in an undergraduate psychology course.

\section{Materials}

The decision task was a computer-simulated card game similar to the game of blackjack, where the goal is to maximize the sum of cards without going over 21 . The modified blackjack task was designed for several purposes. First, it was a simplification of the familiar blackjack game (e.g., in our task, we excluded aces to avoid the ambiguity of whether they should be counted as $1 \mathrm{~s}$ or $11 \mathrm{~s}$ and excluded jacks, queens, and kings to avoid issues relating to perceptual recognition of the face cards; we also did not allow "splitting" and other options that are part of the blackjack game). Second, the probabilities associated with each choice are easily manipulated by the values of the cards dealt to the players, and decisions are easily quantified by the time it takes to make a binary decision (to take an additional card or not).

On each trial, the participant received two cards, which appeared at the top half of the computer screen, and the computer received a single card, which appeared at the bottom half of the screen. Each card was $3.2 \times 4.9 \mathrm{~cm}$, with its number displayed a single time in the center of the frame, using a 36-size font, Geneva typeface.

The player's cards were manipulated to vary the level of risk produced by the probability that the player's total would exceed 21 (and lose the hand). At the very low end of the risk level spectrum were the trials with a sum of 11 or less, in which case there was no risk in taking an additional card (regardless of the value of the additional card, participants could not go over 21). There were also trials with low risk (trials with sums of 12 or 13), trials with medium risk (trials with sums of 14 or 15), trials with high risk (trials with sums of 16 or 17), trials with very high risk (trials with sums of 18 or 19), and trials with infinite risk (trials with a sum of 20 , in which case it is wrong to take an additional card, because participants would necessarily go over 21 and lose their entire hand).

The value showing on the computer's card was also manipulated on each trial. The computer's card was programmed to have a low level (card values of 2,3 , or 4), a moderate level (card values of 5 , 
6 , or 7), or a high level (card values of 8,9 , or 10 ). A high level for the computer card was used to increase the total sum that the player needed to beat the computer and, consequently, increase the player's need to take another card. A low level was used to decrease the total sum that the player needed to beat the computer and, consequently, reduce the player's need to take another card.

Trials were counterbalanced to ensure an equal number of trials with all the possible combinations of player's and computer's cards. For each possible sum of the participants' two cards ( 17 possible sums, from 4 to 20), a group of 9 trials was constructed, giving a total of 153 trials. Each of the 9 trials in every group was matched to one of the nine possible values of the computer's card $(2-10)$. The possible combinations of the participants' cards were also counterbalanced (e.g., for the total sum of 14 , all the following combinations were used as equally as possible: 4 and 10,5 and 9,6 and 8,7 and 7,8 and 6,9 and 5, and 10 and 4). For administering the task, the 153 trials were organized in a sequence of nine blocks, each consisting of 17 trials. Each block contained a single presentation of all the possible sums of cards, and the order of the trials within the blocks was randomized. (Although this counterbalancing procedure has experimental design advantages, it has the disadvantage of influencing the frequencies in such a way that each triple of cards is not equally likely.)

\section{Procedure}

The participants were tested individually in a single testing session. Half the participants were first tested with no time pressure and then with time pressure; the other half of the participants were tested in the reverse order (in each group of participants who were tested in a certain order- either time pressure first or no time pressure first-half the participants were male and half were female). For both the time pressure and the no time pressure conditions, the participants were tested on the identical 153 experimental trials, except that the order of the trials was different (trials were randomized within each block). Time pressure was created by asking the participants to respond as quickly as possible. It was emphasized that their response time was critical. Under the no time pressure conditions, the participants were told to take as much time as they needed to respond and were asked to carefully consider their decisions. The participants were seated approximately $45 \mathrm{~cm}$ from the computer screen. Instructions appeared on the computer screen, followed by three practice trials.

At the onset of each trial, an exclamation point was displayed on the computer screen. When the participants were ready to begin the trial, they pressed the space bar. Three cards appeared in each trial; two were considered to be dealt to the participant, and one to the opponent. On the basis of the value of these cards, the participant decided whether or not to take an additiona! card ("splitting," "insurance," and other options from the game of blackjack were not allowed). If a new card was taken, the value of the new card was added to the participant's total; if the participant decided not to take another card, the total remained unchanged. The participants' goal was to have the sum of their cards be higher than their opponent's, without exceeding 21 .

The participants were instructed to assume that, after they made their decision, they and their opponent would have the opportunity to take additional cards. They were also informed that they would not see what additional card they received, what additional cards the opponent received, or who won the hand (this was done to ensure that participants would not change decision strategy or criterion during the task as a result of recent positive or negative outcomes of previous decisions; see Dror, Rafaely, \& Busemeyer, 1999, and Rafaely, Dror, \& Busemeyer, 1998, for details). Rather, after making a decision, the participants would be presented on the next trial with a new and independent sample of cards. If the participants chose to take an additional card, they pressed the yes key (the " $b$ " key, which was labeled yes). If the participants did not want an additional card, they pressed the no key (the " $n$ " key, which was la- beled no). After the participants responded, a blank screen appeared for $350 \mathrm{msec}$, followed by an exclamation point to signal the beginning of a new trial. The participants responded yes and no by using two fingers of their dominant hand and pressed the space bar with their nondominant hand. The participants did not receive any feedback or additional information. Throughout the instructions and practice trials, the participants were encouraged to ask questions, and clarifications were given by the experimenter as needed. However, no talking was allowed during the actual experiment.

\section{RESULTS AND DISCUSSION}

\section{Empirical Data}

First, choice probabilities (proportion of trials in which an additinnal card was requested) are reported, using the player's risk level $(1=$ no risk, $2=$ low risk, $3=$ medium risk, $4=$ high risk, $5=$ very high risk, $6=$ infinite risk), the computer's card level $(1=$ low level, 2 = moderate level, $3=$ high level), and time pressure (no pressure vs. pressure) as within-subjects factors. Second, response times (pooled across choices) are described, using the same three factors. Finally, conditional response times (computed separately for gambling and not gambling responses) are shown, using risk category (low $=$ risk levels 1,2 , or 3 vs. high = risk levels 4,5 , or 6 ), time pressure (pressure vs. no pressure), and response category (choose to gamble vs. choose not to gamble) as factors. (Computer card was omitted because of sample size limitations.)

Choice probability. As is illustrated in Figure 1 (left panels), choice probability decreases as risk level increases. More important, the curve within each panel for the no time pressure condition is steeper than the corresponding curve for the time pressure condition, producing a crossover interaction between risk level and time pressure. This crossover result confirms the a priori prediction made by sequential sampling models.

A specific test of the critical risk level $\times$ time pressure crossover interaction was performed by computing a single degree of freedom contrast, using the logistic transformed choice proportions as follows. We computed the difference between the no time pressure and time pressure conditions at two different levels of risk, levels 2 and 5 . These two levels were chosen because they are moderately extreme, but not to the extent that the choice probabilities approach zero or one. At level 2, the difference was predicted by the sequential sampling models to be positive, and at level 5 it was expected to be negative. We observed the following results: First, the difference between the no pressure and the pressure conditions for the risk level 2 produced a positive contrast equal to +.56 ; second, the difference between the no pressure and the pressure conditions at risk level 5 produced a negative contrast equal to -.32 ; finally, the contrast of these two differences was significant, according to a $t$ test $[t(155)=10.08, p<.0001]$.

Another notable finding is that the computer's card level moderated the effects of risk level and time pressure: When there was no time pressure, a low level for 
Observed, Computer Card is Low

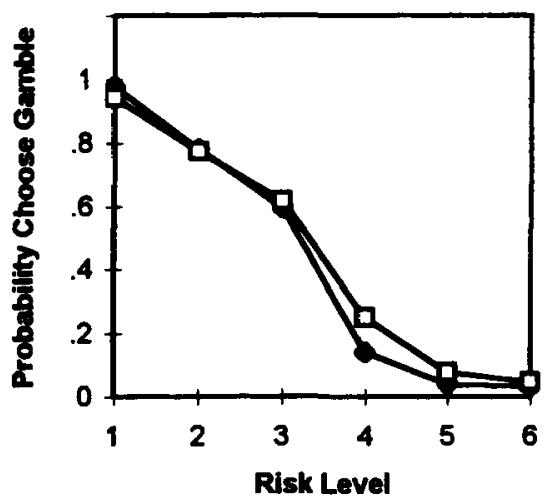

Observed, Computer Card is Med

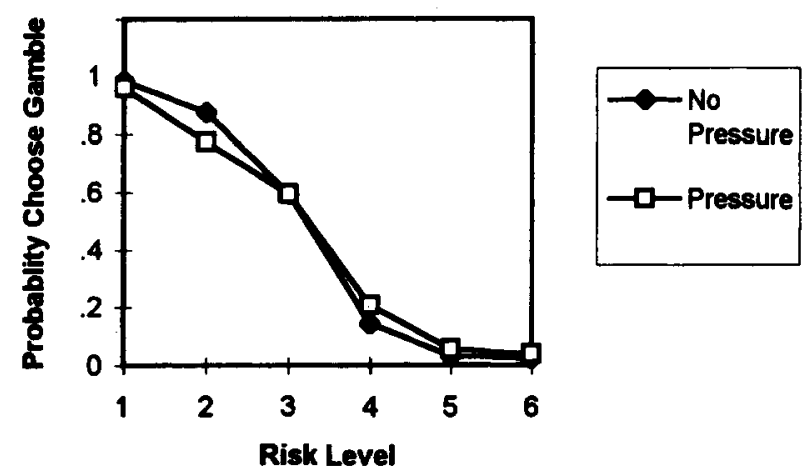

Observed, Computer Card is High

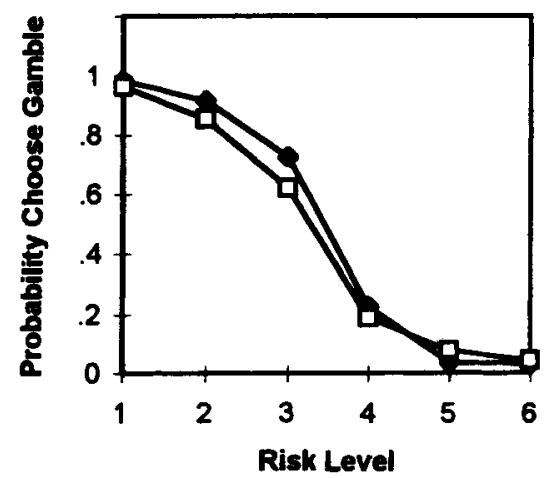

Predicted, Computer Card is Low

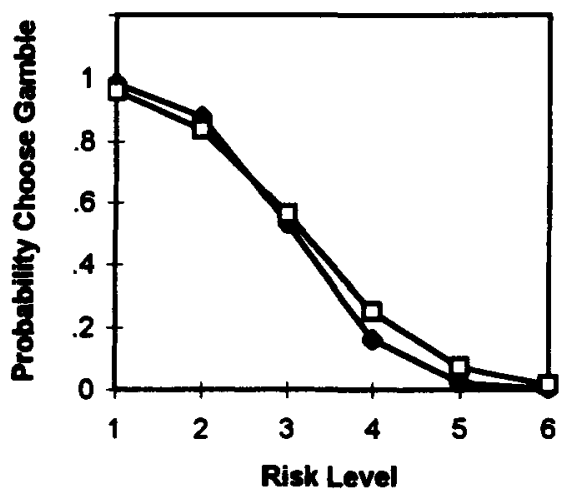

Predicted, Computer Card is Med

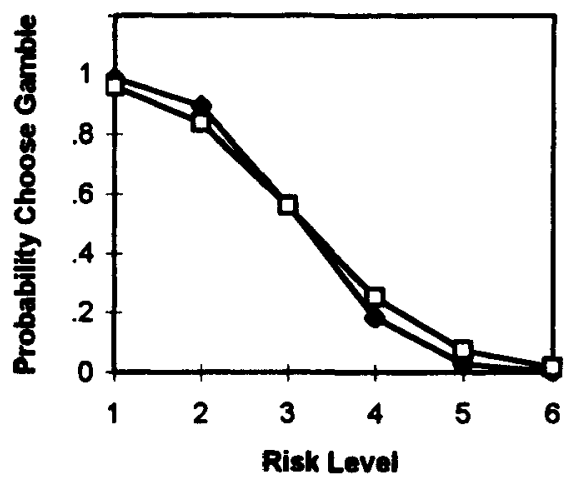

Predicted, Computer Card is High

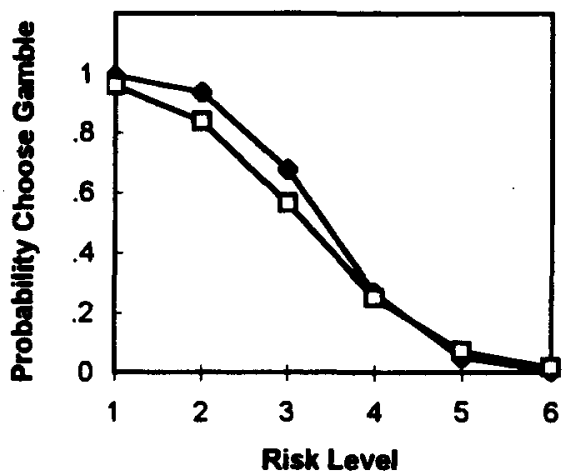

Figure 1. The left three panels show the probability of taking an additional card by the participants as a function of risk level. The top, middle, and bottom panels are for low, moderate, and high computer card levels. In all panels, there is a backward-S-shaped curve as risk level increases, and the time pressure curve is flatter than the no time pressure curve. Furthermore, there is a crossover interaction between the curves for the time pressure and no time pressure conditions in each of the panels. These effects are moderated by the computer card level, but only for the no time pressure condition. The right three panels show the predictions of the model for choice probability under the same experimental conditions. The steeper curve represents the predictions when the inhibitory threshold is set to a high criterion and the bound is increased, and the flatter curve represents the predictions when the inhibitory threshold is set to a low criterion (under time pressure) and the bound is smaller. The precise quantitative form of the curves depends on specific parameter values estimated from the data, but the crossover interaction pattern and the effect of the computer card level is a parameter-free prediction of the model. 
the computer's card suppressed the tendency to gamble at the low risk levels, whereas a high level for the computer's card enhanced the tendency to gamble at the low risk levels; however, when there was time pressure, the computer's card had little or no effect on the choice probability. If the data for the time pressure condition is replotted with choice probability as a function of risk level and a different curve for each computer card level, the three curves lie virtually right on top of each other. This reflects the fact that the computer card level had no effect under the time pressure condition and suggests that the participants did not have sufficient time and resources to consider the computer's card.

The trends in Figure 1 are supported by statistical tests obtained from a three-way repeated measures analysis of variance (ANOVA) using the logistic transformed choice proportion for each subject and condition as the dependent variable. The main effect of risk level was significant $\left[F(5,155)=178.10, M S_{\mathrm{e}}=0.514, p<.0001\right]$, the risk level $\times$ time pressure interaction was significant $\left[F(5,155)=4.68, M S_{\mathrm{e}}=0.061, p<.0005\right]$, and the risk level $\times$ time pressure $\times$ computer card level interaction also was significant $\left[F(10,310)=2.32, M S_{\mathrm{e}}=0.053\right.$, $p=.0121]$. The main effect of computer card level was significant $\left[F(2,62)=57.31, M S_{\mathrm{e}}=0.097, p<.0001\right]$, the computer card level $\times$ risk level interaction was significant $\left[F(10,310)=15.29, M S_{\mathrm{e}}=0.076, p<.0001\right]$, and the computer card level $\times$ time pressure interaction also was significant $\left[F(2,62)=3.29, M S_{\mathrm{e}}=0.055, p=.0440\right]$.

Response time. As is illustrated in Figure 2 (left panels), the mean response time is an inverted-U-shaped function of risk level. However, the level of this curve is much lower and the slope is much flatter under the time pressure condition than under the no time pressure condition. Finally, the peaks of the curve shift from left to right as the computer card level increases from low to high.

The trends in Figure 2 are supported by statistical tests obtained from a three-way repeated measures ANOVA, using the mean response time for each subject and condition as the dependent variable. The main effect of risk level was significant $\left[F(5,155)=20.92, M S_{\mathrm{e}}=1,010,309\right.$, $p<.0001$ ], the risk level $\times$ time pressure interaction was significant $\left[F(5,155)=11.5, M S_{\mathrm{e}}=642,320, p<.0001\right]$, and the risk level $\times$ time pressure $\times$ computer card level interaction also was significant $\left[F(10,310)=1.86, M S_{\mathrm{e}}=\right.$ $243,049, p=.0498]$. The main effect of computer card level was significant $\left[F(2,62)=3.23, M S_{\mathrm{e}}=294,584\right.$, $p=.0465]$, and the computer card level $\times$ risk level interaction was significant $\left[F(10,310)=2.90, M S_{\mathrm{e}}=\right.$ $274,504, p=.0017]$.

Conditional response time. Figure 3 (top panel) shows the mean response times plotted as a function of risk category, with a separate line for each response category and time pressure condition. As can be seen in the figure, there is a crossover interaction between risk category and response category, but only for the no time pressure condition. The crossover interaction under the no time pressure condition can be summarized as follows: The decision to gamble takes less time than the decision not to gamble when the risk category is low, but the decision to gamble takes more time than the decision not to gamble when the risk category is high. Recall from Figure 1 that choice probability follows the opposite pattern: The decision to gamble is more frequent than the decision not to gamble under the low risk category, but the decision to gamble is less frequent than the decision not to gamble under the high risk category. This replicates previous findings that have shown an inverse relation between the probability that an alternative is chosen and the time required to choose it (Busemeyer, 1982; Petrusic \& Jamieson, 1978).

The trends in Figure 3 are supported by statistical tests obtained from a three-way repeated measures ANOVA, using the conditional mean response time for each subject and condition as the dependent variable. The risk category $\times$ time pressure $\times$ response category interaction effect was significant $\left[F(1,17)=5.557, M S_{\mathrm{e}}=271,476\right.$, $p<.05]$. (Note: the denominator degrees of freedom reflect missing observations owing to the fact that some subjects never chose one of the alternatives under some conditions. The analysis with missing cells was computed with an SAS system type III sums of squares; see SAS manual, 1996.)

\section{Sequential Sampling Model Predictions}

The specific sequential sampling decision model used to generate predictions was derived from decision field theory (Busemeyer \& Townsend, 1993; Townsend \& Busemeyer, 1996), which is the only sequential sampling model that has been mathematically formalized specifically for risky decision-making tasks. Hence, it enabled us to generate quantitative as well as qualitative predictions for the results of this experiment. Furthermore, decision field theory has been successful in explaining a wide range of fundamental findings from research on risk-taking decision making (see Busemeyer \& Townsend, 1993).

Figure 4 illustrates the basic process that is assumed to occur within a single trial of this decision task. The trial begins at time $t=0$ with the presentation of the player's two cards and the computer's card; then the player begins the process of deciding whether or not to take another card on this trial. The process begins with an initial preference state, denoted $\mathrm{P}(0)$, which is equal to zero, assuming that the player starts out unbiased (i.e., $P(0)=$ $0)$. Then, from moment to moment, the player imagines and anticipates the positive or negative outcomes of each choice, given the cards that are presented. At one moment, the player may anticipate winning by taking an additional card, strengthening the approach tendency, so that $\mathrm{P}(t+1)-\mathrm{P}(t)>0$. At a later moment, the decision maker may imagine losing the hand by taking an additional card and going over 21 , strengthening the avoidance tendency so that $\mathrm{P}(t+2)-\mathrm{P}(t+1)<0$.

This vacillation in tendencies continues until either the approach tendency becomes strong enough to exceed an inhibitory threshold to trigger the action of requesting an additional card $[\mathrm{P}(t)>+\theta]$ or the avoidance tendency 
Observed, Computer Card is Low

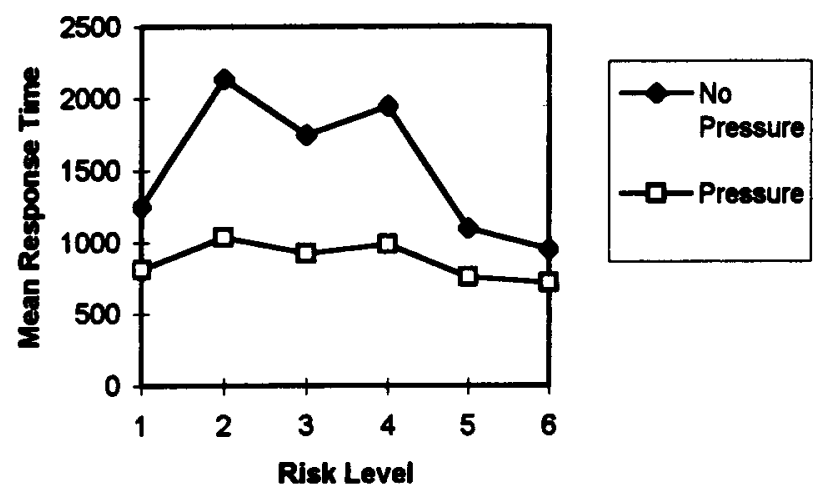

Observed, Computer Card is Medium

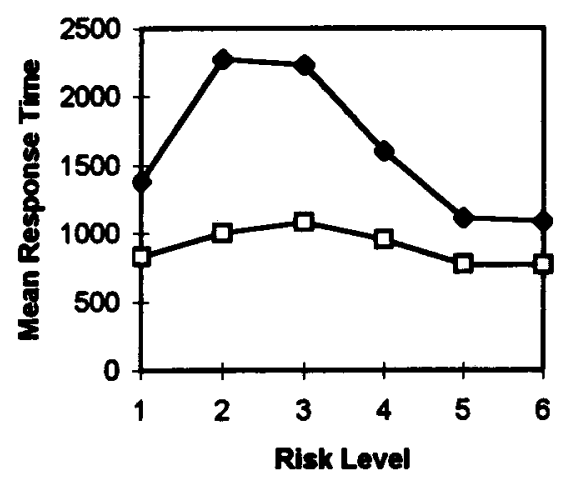

Observed, Computer Card is high

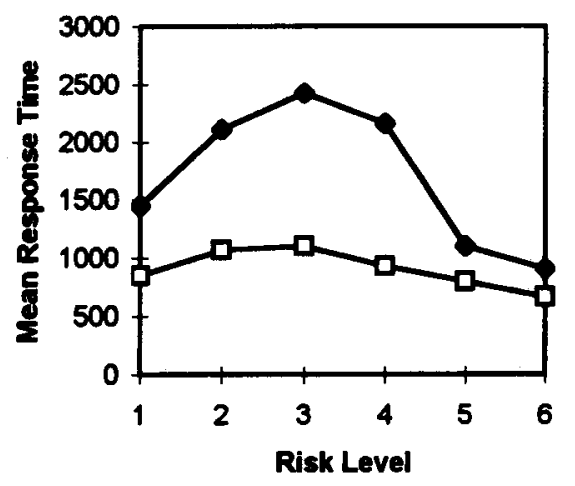

Predicted, Card is Low

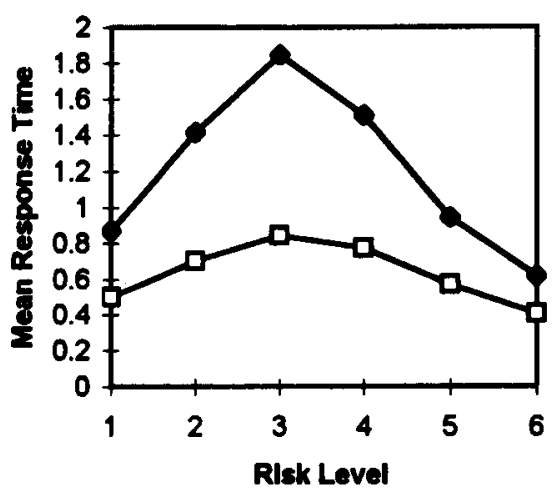

Predicted, Card is Medium

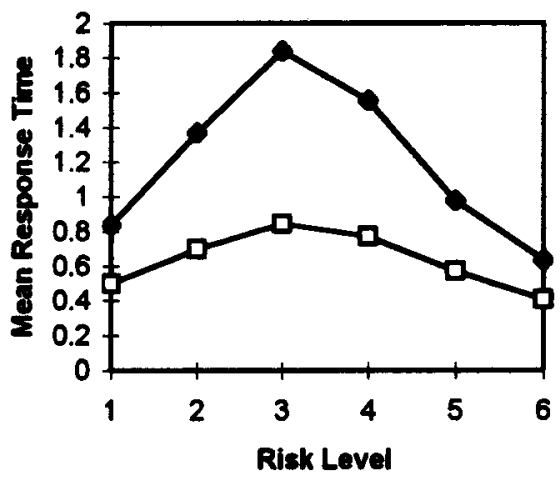

Predicted, Card is High

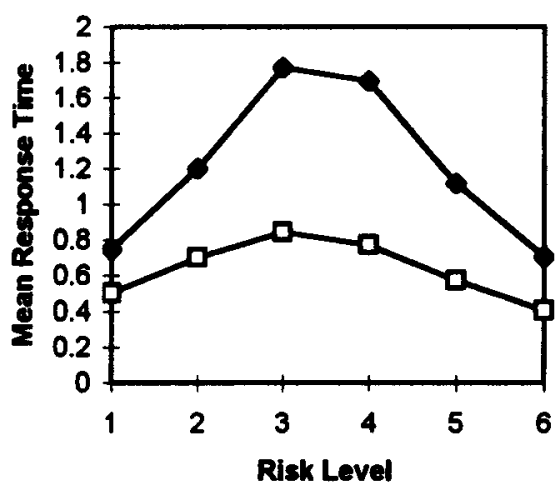

Figure 2. The left three panels show the mean response time (pooled across gambling and not gambling responses) that participants needed to decide whether or not to take an additional card as a function of risk level. The top, middle, and bottom panels are for low, moderate, and high computer card levels. In all panels, the participants needed more time at the difficult trials (with medium levels of risk) and less time at the easy trials (when the risk level was relatively high or low). With time pressure, the inverted-U-shaped response time curve was flatter and lower than the curve with time pressure. The locations of the curves' peaks are influenced by the computer card level. The right three panels show the predictions of the model for mean response time (pooled across responses) for the same experimental conditions. The model accurately predicts the inverted- $U$-shaped relations between mean choice time and risk level for each time pressure condition. 

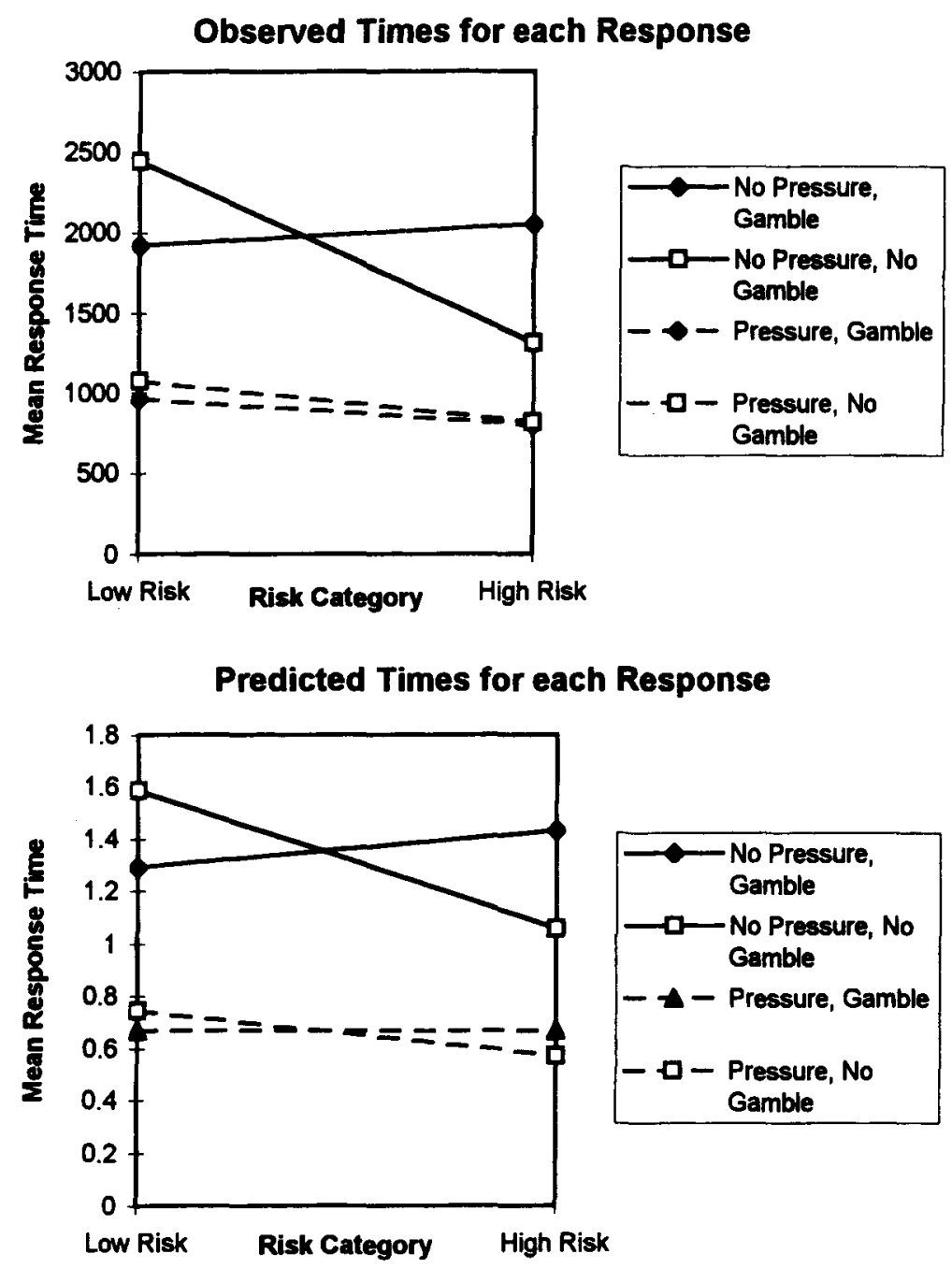

Figure 3. The top panel shows the mean response time participants needed for each response alternative (to take a card and not to take a card-the conditional response time) as a function of risk level. There is a crossover interaction between the two, but only for the no time pressure condition. The bottom panel shows that the model accurately predicts the behavioral data.

becomes strong enough to exceed an inhibitory threshold to trigger the action of declining an additional card $[\mathrm{P}(t)<-\theta]$. The final choice on a particular trial is determined by whether the approach tendency or the avoidance tendency crosses the threshold first. The decision time is determined by the time it takes to exceed the threshold magnitude.

According to the model of the decision process described above, choice and decision time are controlled by two factors: One is $\theta$, the magnitude of the inhibitory threshold; the second is $d$, the mean change in preference state at each moment. The magnitude of the inhibitory threshold, $\theta$, is assumed to be determined by the time pressure manipulation: The threshold is reduced under the high time pressure condition. The inhibitory threshold controls the amount of time spent thinking about the decision, which in turn controls the amount of information accumulated about the anticipated outcomes produced by each action. Increasing the threshold increases the average decision time. Thus, more information is accumulated, which causes greater decision accuracy (the probability of choosing the optimal response that maximizes the likelihood of winning). Decreasing the threshold (under time pressure) reduces the average decision time. Thus, less information is accumulated, which causes decreased decision accuracy. (See Busemeyer, 1985, for a more formal analysis, or the Appendix.)

The sign of the mean change in preference state, $d$, determines whether the approach or avoidance tendency grows stronger on the average over time. If $d$ is positive, the approach tendency (take another card) grows stronger over time than the avoidance tendency, and if $d$ is nega- 


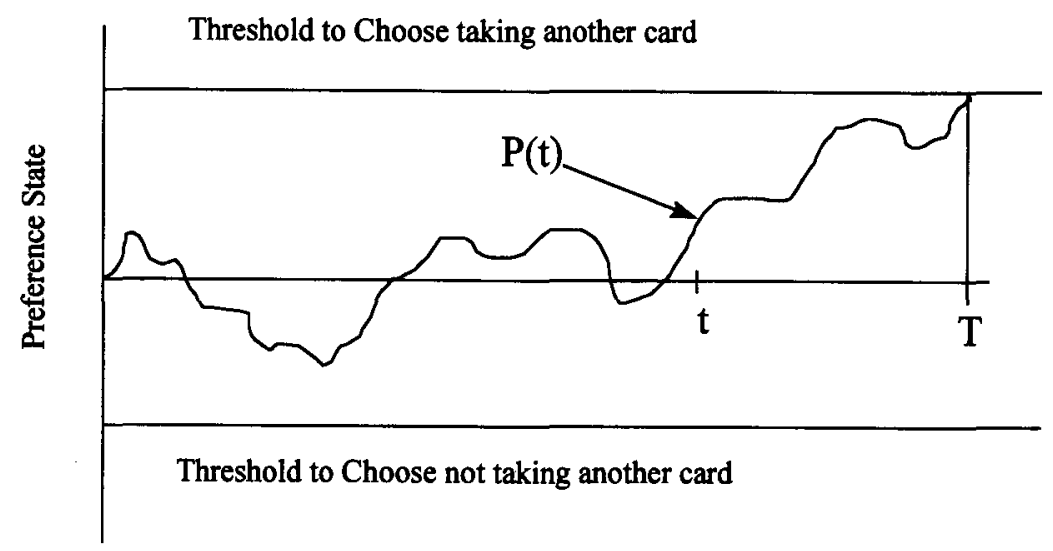

\begin{abstract}
Figure 4. A model of the task based on decision field theory. The horizontal axis represents the time interval, starting with the initial presentation of the two cards, until a final decision is reached on whether or not to take an additional card. The vertical axis represents the participant's relative preference, where a positive preference is a tendency to take another card and a negative preference is a tendency to avoid taking another card. The jagged curve in the figure represents the evolution of the participant's preference state over time, $P(t)$. The action of taking an additional card or not is determined by which threshold is exceeded on that trial, and the decision time is determined by the time interval required to exceed that threshold (the vertical line at time $\boldsymbol{T}$ ). As the bounds decrease (when the thresholds are lowered under time pressure), the time interval to exceed a threshold is smaller.
\end{abstract}

tive, the avoidance tendency (decline another card) grows stronger over time than the approach tendency.

Recall that the probability of exceeding 21 and losing the game by taking another card increases as the player's total increases, but the need to take another card to beat the computer increases as the value of the computer's card increases. These two factors are represented in the model by assuming that the mean change in preference state, $d$, is a decreasing function of the risk level and an increasing function of the computer card level. This implies that, for each fixed value of the computer card level, the probability of choosing to gamble will be a decreasing function of the risk level (see the Appendix for details). However, under the time pressure condition, there may be insufficient time to consider the computer's card, so in this case, the computer's card is ignored. (Changes in attention over time are consistent with Diederich's [1995] multiattribute generalization of decision field theory.)

The mathematics used to derive the formulas for this particular model have been presented elsewhere (Busemeyer \& Townsend, 1992, 1993), and the Appendix provides the derived formulas used to compute the predictions shown below. It is important to note that strong quantitative tests of the model are made possible through the use of both choice probability and choice response time measures of preference. The basic method for testing the model is first to estimate the model parameters from the choice probability data and then to use these same parameters to generate parameter-free predictions for the mean response time data.
More specifically, seven model parameters were estimated from the 36 observed choice proportions, and these parameter estimates were used in the formulas to compute the choice probabilities from the model. The critical test is obtained by entering these same seven parameters into the formulas for computing the predictions for choice response time data. This provides a generalization test of the model by producing predictions for all of the response time data that do not require estimating any new parameters.

First, the right-hand panels of Figure 1 illustrate the fits to the choice probability data. As can be seen by comparing the left and the right panels, the model correctly reproduces the crossover interaction between risk level and time pressure, and it also reproduces the effects of computer card level on choice probability. Although the specific form of the curves producing the crossover interaction depends on the estimated parameters, the backwardS-shaped form of the function, as well as the crossover interaction, is a necessary property of the model. In particular, if the data failed to show this crossover, the model would be unable to fit the results. ${ }^{1}$

Second, the right-hand panels of Figure 2 illustrate the predictions for the response time data. Note that these predictions are made without using any parameters (this includes the time unit for the predictions, so only the pattern of the predictions is important). As can be seen by comparing the left and the right panels, the model correctly predicts the inverted-U-shaped function relating response time and risk level, and the model also correctly 
predicts the proportional reduction in response time at each risk level produced by the time pressure manipulation. Finally, the model correctly predicts the shift in the peak of this U-shaped function with increases in the computer card level.

A strong test of the model is obtained by evaluating the predictions for decision times conditioned on the specific choice (the response chosen). The model correctly predicts that the mean time to take another card is shorter than the mean time to choose to not take another card, when the risk level is relatively low. The model also correctly predicts the opposite order when the risk level is relatively high. Furthermore, the model predicts that this crossover interaction effect is attenuated by time pressure (see the bottom part in Figure 3).

\section{Strategy-Switching Model Predictions}

An alternative explanation for the effects of time pressure on decision behavior is that subjects switch to simpler heuristic strategies under time pressure. Two possi-
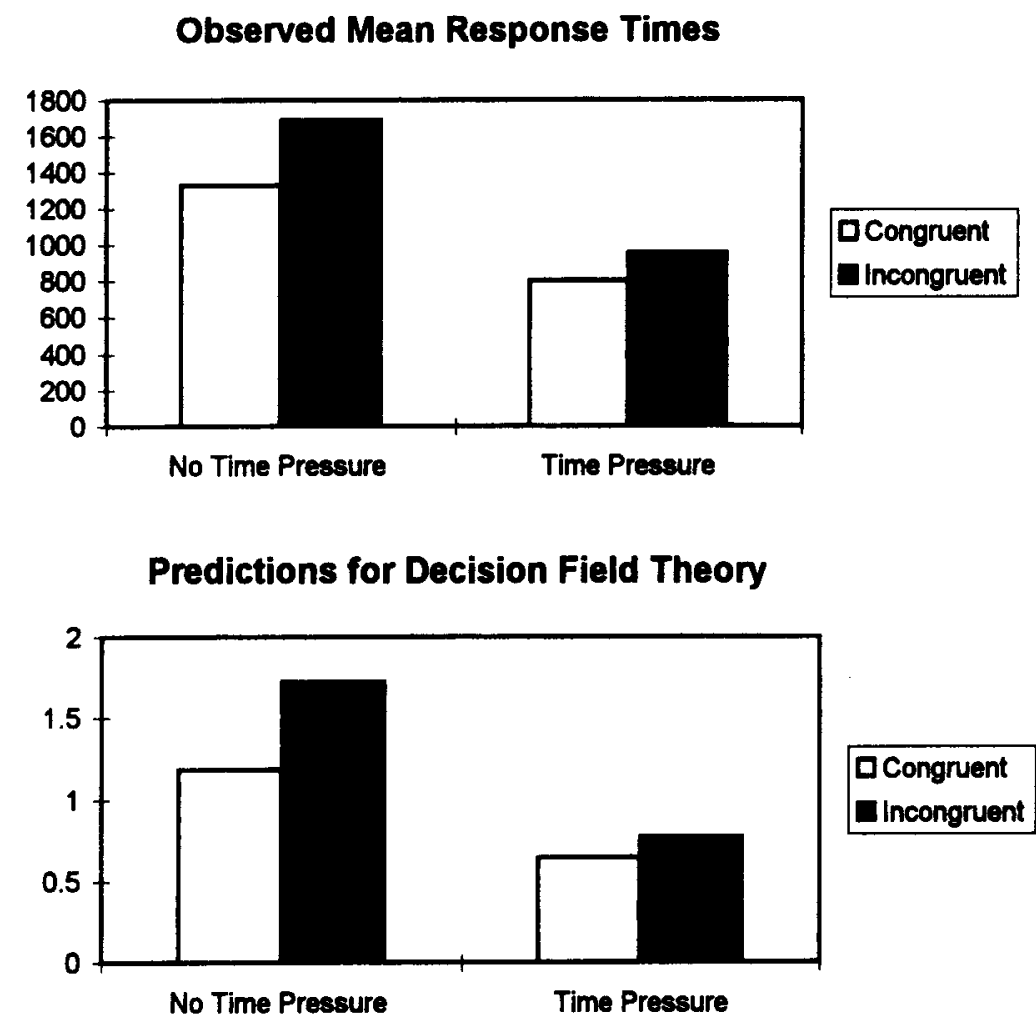

\section{Predictions for Fast Guess Model}

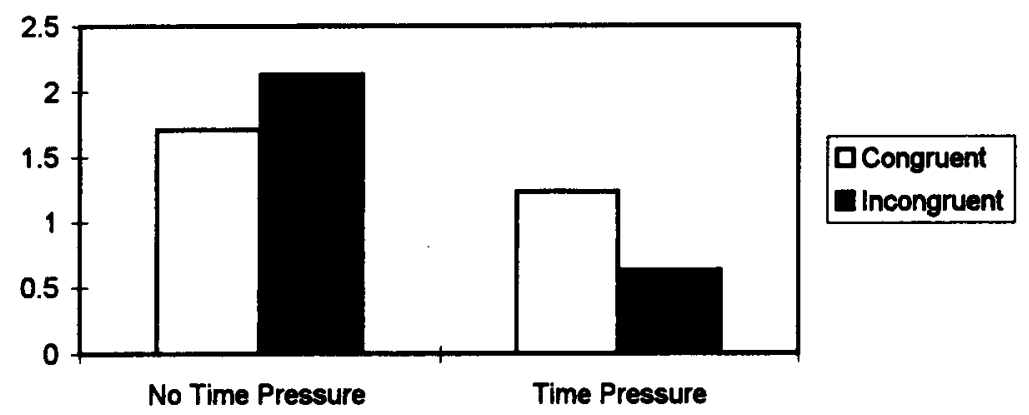

Figure 5. The unfilled bars represent the mean response time for a congruent response, and the dark filled bars represent the mean response time for the incongruent response. The first pair represents the no time pressure condition, and the second pair represents the time pressure condition. The top panel represents the observed data, the middle panel represents the predictions of the original sequential sampling model, and the bottom panel represents the predictions of the fast-guess model. 
bilities were discussed earlier, the never-bust strategy and the fast-guess strategy. The crossover interaction effect observed in Figure 1 clearly rules out the never-bust strategy, but it does not rule out the fast-guess strategy. A critical test of the fast-guess hypothesis can be obtained by examining the predictions for congruent and incongruent mean response times. In general, the fast-guess model predicts fast incongruent responses, whereas the original sequential sampling model predicts slow incongruent responses (see the Appendix for details).

More specifically, the fast-guess model asserts that, under time pressure, participants will make a fast random guess on some proportion of trials. To test this model, new parameters were estimated from the sequential sampling model by fitting it to the choice data and mean response time data with one major change (see the Appendix for details). Instead of decreasing the threshold bound under time pressure conditions, this parameter was held constant, and a new parameter was added, representing the probability of fast guessing under time pressure. In sum, seven parameters (six original plus one fast guess) were estimated from the choice probability data, and these same parameters were used to make predictions regarding decision time. This model produced essentially the same fit to the choice probability data as the original sequential sampling model.

The critical test of the sequential sampling model versus the fast-guess model is shown in Figure 5. The top panel shows the observed mean time to make congruent (white bar) versus incongruent (dark bar) choices, where a congruent choice was to take another card under very low risk (levels 1 or 2) and an incongruent choice was the decision not to take a card under these same conditions. ${ }^{2}$ The left pair of bars represents the no time pressure condition, and the right pair represents the time pressure condition. Note that the incongruent responses were made more slowly than the congruent responses under the critical time pressure condition. ${ }^{3}$

The middle panel shows the corresponding predictions of the original sequential sampling model. As can be seen in the figure, the original sequential sampling model accurately predicts the pattern of observed results under both time pressure conditions. The bottom panel shows the corresponding predictions produced by the fast-guess model. As can be seen, the fast-guess model fails to describe the pattern of results under the time pressure condition. In sum, the finding of slower incongruent responses, as compared with congruent responses, is contrary to the fast-guess model and supports the original sequential sampling model.

\section{GENERAL DISCUSSION}

Recent emphasis has been placed on the decision process in numerous successful models from a variety of cognitive domains (Link, 1992; Nosofsky \& Palmeri, 1997; Ratcliff, 1978; Smith, 1995). It seemed interesting to examine this decision process in the more "pure" decision-making domain. This is especially important inasmuch as the above-mentioned models all have similar conceptualizations of what constitutes the decisionmaking process - namely, sequential sampling over time until a certain threshold is met.

We collected behavioral data on a risk-taking decisionmaking task (Dror et al., 1998) to independently test these types of models. Because sequential sampling models have considered the effects of time pressure (Link, 1992; Nosofsky \& Palmeri, 1997; Ratcliff, 1978), we included a time pressure component in our behavioral task. We used decision field theory (Busemeyer \& Townsend, 1993; Townsend \& Busemeyer, 1996) to generate predictions, because it is a sequential sampling model that has been mathematically developed for risky decision making and, thus, it enabled us to make precise predictions for our behavioral gambling-like task.

Our behavioral data were consistent with previous behavioral research (Dror et al., 1998), showing that participants systematically were less likely to take a card as the risks increased and that response time for making the decisions decreased as the risks were toward the ends of the risk level spectrum. Our manipulation of time pressure caused a polarization effect; participants were more conservative and less likely to take an action (request an additional card) at the lower levels of risk and were more risky and likely to take an action at the higher levels of risk. That is, the S-shaped choice probability curve, as a function of risk, was flatter under the time pressure condition. Response times decreased with time pressure, and the inverted-U-shaped choice time curve, as a function of risk, was also flatter under the time pressure condition. Finally, the time to make a choice was inversely related to the probability of making that choice, replicating previous findings (Busemeyer, 1982; Petrusic \& Jamieson, 1978).

The empirical results described above were consistent with the predictions generated by decision field theory. The theoretical analyses based on this model indicated that time pressure produced two different effects on the decision process. One was to reduce the threshold bound for the cumulative preference strength needed to make a decision, and the second was to reduce the amount of attention given to less important dimensions in the task. The effect of time pressure on the threshold bounds is consistent with a long-standing assumption of decision field theory (Busemeyer, 1985). The effect of time pressure on attention to less important dimensions is consistent with recent generalizations of decision field theory to multidimensional choice problems (Diederich, 1995).

An alternative hypothesis, based on the idea of strategy switching, was also evaluated. Many recent studies (for an overview, see Svenson \& Maule, 1995) have attempted to explain changes in choice behavior under time pressure by changes in decision strategies, from a more accurate and time-consuming strategy to a less accurate but simpler and faster heuristic strategy (see, e.g., Johnson et al., 1995; Payne et al., 1996). Keren and Wagenaar 
(1985) have identified two such strategies in blackjacktype tasks (see, also, Anderson \& Brown, 1984; Bond, 1974; Phillips \& Amrhein, 1989). First, the basic strategy, which is the optimal strategy that maximizes the probability of winning, requires the player to consider his or her own cards as well as the computer's card. Second, the never-bust strategy is a conservative tendency to avoid taking an additional card and to stay with the current total. Note that the never-bust strategy only requires attention to the player's cards and does not require attention to the computer's card. Although nonoptimal, the never-bust strategy is attractive because it is simple and, more important, avoids the possibility of actively taking an action (requesting an additional card) that may be directly responsible for losing the game.

The basic or optimal strategy for our risk-taking task is to always choose to take an additional card if the current total sum of the cards falls below a cutoff criterion (this criterion depends on, among other things, the computer's card). In other words, if we were to plot the choice probability curve predicted by this strategy (similar to that shown in Figure 1), it would be a backward step function with an abrupt jump from one to zero at the cutoff. However, if this cutoff varies across participants, the curve produced by averaging across participants would appear as a backward-S-shaped curve like that shown in Figure 1.

The never-bust strategy is a very simple heuristic strategy of not taking an additional card if there is some risk that the player's new total will exceed 21 . It is reasonable to expect that, under time pressure, at least on some proportion of trials, participants may abandon the more difficult process of locating the optimal cutoff for the simpler heuristic strategy that guarantees not losing the entire hand by taking an additional card. However, this strategyswitching model does make one important distinguishing prediction. That is, the curve produced under time pressure must lay completely below that predicted by no time pressure (because the proportion of trials in which participants use the simpler strategy of not taking an additional card always increases under time pressure). However, as is apparent in Figure 1 (top panel), our data do not support this prediction.

Another plausible heuristic strategy is to resort to fast random guesses under time pressure. This fast-guess model explains the time pressure $\times$ risk level crossover interaction effect on choice probability as being the result of mixing random guesses on some proportion of trials, which flattens out the backward-S-shaped curve. However, the fast-guess model incorrectly predicts that subjects will make faster incongruent, as opposed to congruent, responses under time pressure, which is contrary to the observed results.

In sum, for this particular task, the never-bust and fastguess strategies are the most common and obvious heuristics, and we can rule out the hypothesis that participants tend to switch to these particular strategies under time pressure. In contrast, the sequential sampling model provides a comprehensive explanation of both the choice probability and the choice response time behavioral data.

Of course, it is impossible to enumerate all conceivable strategies, so we cannot completely eliminate the general form of this explanation. Moreover, one could interpret changes in attention to the opponent's card as a kind of strategy switch. But then the notion of strategy becomes so broad that one has to question its testability or predictive utility.

One final comment is that our results do not rule out other possible effects of time pressure on decision processes, such as a speed-up of each processing step (see Ben Zur \& Breznitz, 1981). But note that simply speeding up processing by a constant factor under time pressure cannot explain the changes in the pattern of choice probabilities and response times produced by time pressure.

It is interesting to note that our empirical results, obtained from a traditional decision-making task, appear to be very similar to the speed-accuracy tradeoff results found in other cognitive tasks, such as perception (see Link, 1992), memory (see Ratcliff, 1978), and categorization (see Nosofsky \& Palmeri, 1997). Furthermore, the success of sequential sampling models in accounting for the results obtained from this risk-taking decisionmaking task strengthens our confidence in the general use of this decision process across a variety of cognitive tasks (for further discussion on the usefulness of such models, see Dror \& Gallogly, 1999). In summary, both the empirical results and the theoretical analyses presented in this study support the view that common principles of decision processes underlie a wide range of cognitive tasks.

\section{REFERENCES}

Albert, D., Aschenbrenner, K. M., \& Schmalhofer, F. (1989). Cognitive choice processes and the attitude-behavior relation. In A. Upmeyer (Ed.), Attitudes and behavioral decisions (pp. 61-99). New York: Springer-Verlag.

ANDERSON, G., \& BROWN, R. I. (1984). Real and laboratory gambling, sensation-seeking and arousal. British Journal of Psychology, 75, 401-410.

Aschenbrenner, K. M., Albert, D., \& Schmallofer, F. (1986). Stochastic choice heuristics. Acta Psychologica, 56, 153-166.

Ben Zur, H., \& BreznitZ, S. J. (1981). The effect of time pressure on risky choice behavior. Acta Psychologica, 47, 89-104.

BoND, N. (1974). Basic strategy and expectation in casino blackjack. Organizational Behavior \& Human Performance, 12, 413-428.

BuSEMEYER, J. R. (1982). Choice behavior in a sequential decision making task. Organizational Behavior \& Human Performance, 29, 175-207.

BuSEMEYER, J. R. (1985). Decision making under uncertainty: A comparison of simple scalability, fixed sample, and sequential sampling models. Journal of Experimental Psychology: Learning, Memory, \& Cognition, 11, 538-564.

BuSEMEYER, J. R., \& TownsEnd, J. T. (1992). Fundamental derivations for decision field theory. Mathematical Social Sciences, 23, 255-282.

Busemeyer, J. R., \& Townsend, J. T. (1993). Decision field theory: A dynamic-cognitive approach to decision making in an uncertain environment. Psychological Review, 100, 432-459.

DiEDERICH, A. (1995). A dynamic model for multi-attribute decision problems. In J. Caverni, M. Bar-Hillel, F. Hutton-Barron, \& H. Junger- 
mann (Eds.), Contributions to decision making (pp. 75-191). Amsterdam: Elsevier.

Dror, I. E., \& Gallogly, D. P. (1999). Computational analyses in cognitive neuroscience: In defense of biological implausibility. Psychonomic Bulletin \& Review, 6, 173-182.

Dror, I. E., Katona, M., \& Mungur, K. (1998). Aging and decision making: To take a risk or not? Gerontology, 44, 67-71.

Dror, I. E., RAFAely, V., \& Busemeyer, J. R. (1999). The dynamics of decision making as a function of recent outcomes and possible consequences. Paper presented at the Sixth European Congress of Psychology, Rome.

Goldstein, W. M., \& Weber, E. U. (1996). Content and discontent: Indications and implications of domain specificity in preferential decision making. In J. R. Busemeyer, D. L. Medin, \& R. Hastie (Eds.), The psychology of learning and motivation: Vol. 32. Decision making from a cognitive perspective (pp. 83-126). New York: Academic Press.

Johnson, E. J., Payne, J. W., \& Bettman, J. R. (1995). Adapting to time constraints. In O. Svenson \& J. Maule (Eds.), Time pressure and stress in human judgment and decision making (pp. 167-178). New York: Plenum.

KerEN, G. B., \& WAGENAAR, W. A. (1985). On the psychology of playing blackjack: Normative and descriptive considerations with implications for decision theory. Journal of Experimental Psychology: General, 114, 113-158.

KORNBROT, D. E. (1989). Random walk models of binary choice: The effect of deadlines in the presence of asymmetric payoffs. Acta Psychologica, 72, 103.

LINK, S. W. (1992). The wave theory of difference similarity. Hillsdale, NJ: Erlbaum.

Nosorsky, R. M., \& PALMER, T. J. (1997). An exemplar based random walk model of speeded classification. Psychological Review, 104, 266-300.

Payne, J. W., Bettman, J. R., \& Luce, M. F. (1996). When time is money: Decision behavior under opportunity-cost time pressure. Organizational Behavior \& Human Decision Processes, 66, 131-152.

Petrusic, W. M., \& Jamieson, D. G. (1978). Relation between probability of preferential choice and time to choose changes with practice. Journal of Experimental Psychology: Human Perception \& Performance, 4, 471-482.

Phillips, J. G., \& Amrhein, P. C. (1989). Factors influencing wagers in simulated blackjack. Journal of Gambling Behavior, 5, 99-111.

RAFAELY, V., DroR, I. E., \& BusEMEYER, J. R. (1998). The susceptibility of young and old adults to positive and negative outcomes of recent decisions. Abstracts of the Psychonomic Society, 3, 41.

RATCLIFF, R. (1978). A theory of memory retrieval. Psychological Review, 85, 59-108.

RATCLIFF, R., VAN ZANDT, T., \& McKoon, G. (in press). Connectionist and diffusion models of reaction time. Psychological Review.

SMITH, P. L. (1995). Psychophysically principled models of visual simple reaction time. Psychological Review, 102, 567-593.

SVENSON, O., \& MAULE, J. (1995). Time pressure and stress in human judgment and decision making. New York: Plenum.

TOWNSEND, J. T., \& BuSEMEYER, J. R. (1996). Dynamic representation of decision-making. In R. F. Port \& T. van Gelder (Eds.), Mind as motion: Explorations in the dynamics of cognition. Cambridge, MA: MIT Press.

WALLSTEN, T. S. (1995). Time pressure and payoff effects on multidimensional probabilistic inference. In O. Svenson \& J. Maule (Eds.), Time pressure and stress in human judgment and decision making (pp. 167-178). New York: Plenum.

\section{NOTES}

1. Note that the above model assumes that time pressure has two separate effects on the decision process. One is a decrease in the threshold bound, and the second is a decrease in attention to the opponent's card. One might question whether both of these assumptions are really necessary. To answer this question, the model was refit under two different constraints. On the one hand, when the model was refit assuming a decrease in threshold but no decrease in attention under time pressure, the model failed to produce the observed time pressure $X$ opponent's card interaction effect. On the other hand, when the model was refit assuming a decrease in attention but no decrease in threshold under time pressure, the model failed to produce the observed crossover interaction between time pressure and risk level. In sum, both assumptions were required to explain the choice probability results.

2 . The two extreme low risk level conditions were used for this test for two reasons. The first reason is that the two most extreme levels provide the most diagnostic and the clearest definitions for congruent and incongruent responses. Second, the experimental design provided 90 trials per subject under the two lowest levels, but it provided only 18 trials per subject under the two highest levels. The reader should also note that the data shown in Figures 3 and 5 overlap but are not the same. For example, in Figure 3, the open square plotted under the low risk category represents mean time to choose no card, averaged over risk levels 1,2 , and 3 ; in Figure 5, the black bar above the time pressure label represents the mean time to choose no card, averaged over risk levels 1 and 2. Risk level 3 was included in Figure 3 in order to include all of the data in the basic analyses. Risk level 3 was not included in Figure 5 because this risk level was too high to treat choosing no card as an incongruent response. In other words, risk level 3 does entail some risk, which makes it an ambiguous case for defining the choice of no card as an incongruent response.

3. A dependent $t$ test was used to perform a statistical test of the hypothesis implied by the fast-guess model. Define $\mu_{\mathrm{C}}$ and $\mu_{\mathrm{I}}$ as the population mean response times for the congruent and incongruent responses, respectively, under time pressure. To falsify the fast-guess model, we need to reject the directional hypothesis $\mathrm{H}_{0}:\left(\mu_{\mathrm{I}}-\mu_{\mathrm{C}}\right)<0$. The sample mean difference between congruent and incongruent response times under time pressure produced a $t$ statistic that exceeded the conventional cutoff for rejecting this hypothesis $[t(31)=1.8, p<.05]$.

\section{APPENDIX}

The purpose of this appendix is to present the formulas used to compute the predictions from the model derived from decision field theory for this task. Decision field theory has seven stages, but it was sufficient to use only stage three (Equations 3c and 3d in Busemeyer \& Townsend, 1993) for this simple task. In this case, the formula for the probability of choosing to gamble for an individual, denoted $\operatorname{Pr}[G]$, derived from the general theory for this task, is

$$
\operatorname{Pr}[G]=1 /[1+\exp (-2 \theta d)] \text {. }
$$

Note that this is an S-shaped logistic function of the mean change in preference state, $d$, and that the threshold, $\theta$, moderates the slope of this logistic function. Recall that $d$ is inversely related to the risk level, and therefore, choice probability is predicted to be a backward-S-shaped function of risk level. Increasing the time pressure decreases $\theta$, which decreases the slope of the logistic function of $d$, and this produces the predicted crossover interaction.

The mean time to make a choice for an individual subject, denoted $E[T]$, derived from the general theory for this task, is

$$
E[T]=(\theta / d)(2 \operatorname{Pr}[G]-1) .
$$

Conceptually, the ratio $(\theta / d)$ can be interpreted as the wellknown formula, travel time equals distance divided by rate of travel. Under time pressure, the distance to travel $\theta$ decreases, so that the time to travel also decreases. Note that this equation does not include a time unit for the decision process, and so the time constants for the predicted and observed times are not equated. Thus, the predicted times are only proportional to the observed times.

Fitting the choice probability data entailed estimating seven parameters (detailed below), by searching for values that min- 
imized the sum of squared deviations between the predicted and the observed choice probabilities. Two of the seven parameters were used to estimate two threshold values $(\theta=1.44$ for the no time pressure condition, and $\theta=0.955$ for the time pressure condition).

Four parameters were used to determine the mean change in preference, $d$, for each of the 36 conditions, as follows. Denote $d_{i j k}$ as the mean change in preference under risk level $i(i=1$, $2,3,4,5,6)$, computer card level $j(j=1,2,3)$, and time pressure condition $k(k=0,1)$. The following expression was used to determine the mean change:

$$
d_{i j k}=\left[(1-k) c_{j}-a(i-3.5)\right] .
$$

When $k=0$ (no time pressure), the mean change equals the difference between the effect of the computer card level and the effect of the risk level; when $k=1$ (time pressure present), the effect of the computer card is eliminated, and the mean change is inversely related to the effect of the risk level. The effect of risk level was assumed to be proportional to the risk level, with the constant of proportionality estimated from the choice data ( $a=$ .842 ). Three parameters were estimated from the choice data to represent the effect of the computer card level $\left(c_{1}=2.85, c_{2}=\right.$ $2.92, c_{3}=3.14$ ).

An additional seventh parameter was used to incorporate individual differences into the model. This was accomplished by adding a random effect $S_{l}$ to Equation 3:

$$
d_{i j k l}=\left[(1-k) c_{j}-a\left(i-3.5+S_{l}\right)\right]
$$

Conceptually, negative values of $S_{l}$ represent individuals that are more risk seeking than the average person and positive values of $S_{l}$ represent individuals who are more risk aversive than the average person. In general, the distribution of individual difference effects is unknown. For simplicity, the individual difference effects $S_{l}$ were represented by a binomial distribution across the values $\{-1.5,-1.0,-0.5,0,0.5,1.0,1.5\}$ with a parameter $p=.42$ (estimated from the choice data). Specifically, the probability of $S_{l}$ was computed by

$$
P\left[S_{l}=s\right]=[(6 !) /(2 s+3) !(3-2 s) !] p^{2 s+3}(1-p)^{3-2 s} \text {. }
$$

To summarize, the choice probabilities and mean response times were computed separately for each condition and each value of the individual difference effect $S_{l}$, using Equations 1, 2 , and 4 , and then these predicted values were averaged across the individual differences $S_{l}$ for each condition, using the probabilities defined by Equation 5 . The seven parameters were estimated with the choice probability data alone, and then these same seven parameters were used to compute the mean response times shown in Figure 3 and Figure 5.
The fast-guess model is obtained by employing Equations 1 and 2 directly for the no time pressure conditions and by modifying Equations 1 and 2 by mixing a guessing probability, $g$, under time pressure:

$$
\operatorname{Pr}[G]=(1-g) /[1+\exp (-2 \theta d)]+(.5) g,
$$

and

$$
E[T]=(1-g)(\theta / d)(2 \operatorname{Pr}[G]-1)+g \times T_{g},
$$

where $T_{g}$ is the mean time required to make a fast guess. The mean time to make a fast guess cannot be estimated from the choice data alone. Therefore, it was necessary to estimate eight parameters from both the choice and the mean response time data: a threshold, $\theta$, constant across time pressure conditions; a guessing rate $g$ under time pressure; three coefficients for the computer card; two coefficients, $a$ and $p$, described earlier, used to determine the mean change for each condition and individual; and, finally, the mean time to make a guess, $T_{g}$. These eight parameters were then used to compute the mean times to make congruent and incongruent responses, shown in Figure 5.

As Figure 5 shows, the predictions computed from the decision field model indicate that the mean response time under very low risk is slower for incongruent responses than for congruent responses for both time pressure conditions. The conceptual reason for this prediction is that, when the risk level is very low, incongruent responses tend to be chosen when the mean change in preference for an individual (Equation 4) is small in magnitude; when this occurs, the rate of travel (see Equation 2) slows down the time reach the boundary for both time pressure conditions. For example, when the risk level is very low but an individual is very risk aversive, the mean change in preference will be close to zero; this is the case that is most likely to produce an incongruent response, but this response also tends to take more time because of the slow rate of travel.

In contrast, the predictions computed from the fast-guess model indicate that incongruent responses are faster than congruent responses under time pressure and very low risk. The conceptual reason for this prediction is that the fast-guess model assumes that a change in decision strategy occurs under time pressure. Thus, it predicts the same result as the decision field model with no time pressure. But under time pressure, the incongruent responses tend to be produced by the fast guesses, which makes the incongruent responses faster than the congruent responses (the latter tend to occur after waiting to reach the criterion bound).

(Manuscript received March 31, 1998; revision accepted for publication July 6,1998 .) 